\title{
Die pleistozäne Vergletscherung und die Frage der Landsenkung im Bereich des Chonos-Archipels/Südchile
}

\author{
Von Klaus Fischer, Augsburg
}

Mit 2 Abbildungen

\begin{abstract}
$\mathrm{Z}$ u s a m m e n f a s s ung. Im Südsommer 1973 wurde das Gebiet der Guaitecas-Inseln und des Chonos-Archipels in Südchile untersucht. Die in der Literatur weitgehend vertretene Ansicht einer Kippung oder Landsenkung der Westseite der Patagonischen Anden als Ursache des „ertrunkenen " Tälerreliefs erweist sich als unzutreffend. Erstmalig können für dieses Gebiet Ausmaß und Reichweite der Vergletscherung angegeben werden.
\end{abstract}

A bstract. In summer 1973 (southern hemisphere!) the Guaitecas-Isles and the ChonosArchipelago in southern Chile were studied. Tilting or subsidence of the western part of the Patagonian Andes have assumed to be at the origin of the "drowned" valleys, we find there, is proved to be uncorrect. For the first time, the extension of the glaciations can be given for the southern part of the Patagonian Andes from $43^{\circ} 70^{\prime}$ to $46^{\circ}$.

Während die Reichweite der pleistozänen Vergletscherung auf der Ostseite der Anden zwischen $41^{\circ}$ und $48^{\circ}$ Süd und auf der W-Seite des Gebirges auf der Insel Chiloë und in der nördlich anschließenden Chilenischen Längstalung im allgemeinen gut bekannt ist (Caldenius 1932, Czajka 1957, Weischet 1970), besteht südlich der Boca del Guafo und des Golfo Corcovado noch eine Kenntnislücke. Auf einer Fahrt mit dem kleinen Schiff "Guamblin“ des Instituto de Investigaciones Geologicas war es mir im Südsommer 1973 möglich, größere Gebiete der Guaitecas-Inseln und des Chonos-Archipels kennenzulernen ${ }^{1}$ ).

Die Guaitecas-Inseln und der Chonos-Archipel zwischen $47^{\circ} 70^{\prime}$ und $46^{\circ}$ Süd (Abb. 1) stellen einen durch zahlreiche Kanäle, auch Esteros oder Carreras genannt, und Buchten in viele Inseln und Halbinseln aufgelösten Teil der Küstenkordillere dar. Vom Festland wird diese Insel- und Halbinselflur durch die Fortsetzung der Chilenischen Längssenke, den Canal Moraleda und die sich anschließenden Kanäle Costa/Estero Elefantes und Errazuriz getrennt. Zwischen den letztgenannten Kanälen stellt die Insel Traiguén das erste große Relikt dieses Grabens über dem Meeresniveau südlich vom Golf von Ancud dar. Die überwiegend von NNW nach SSO gerichteten Kanäle, die der jüngeren Schieferungsrichtung der hier aus Metamorphiten aufgebauten Küstenkordillere folgen, und andererseits die von $\mathrm{O}$ nach W verlaufenden Kanäle gliedern die Landmasse in mehrere große Inselblöcke auf. Die Ost-West-Kanäle sind dabei häufig die Fortsetzung der großen Transversaltäler des Festlandes, z. B. Cisnes-Tal - Canal Puyuguapi - Canal Niñualac und Canal Goñi (südlich bzw. nördlich der Insel James) oder Simpson Tal - Seno bzw. Estero de Aysén Carrera del Cuchi und Canal Darwin südlich der Insel Victoria oder Tal des Rio Huemules - Paso Tres Cruzes - Canal Chacabuco südlich der Inseln Traiguén und Humos.

Die zahlreichen Kanäle, Buchten und Inseln sind das Ergebnis der kräftigen glazialen Überprägung eines präpleistozänen Tälerreliefs. Spuren dieser Überprägung, wie übersteilte Talhänge, Rundhöckerfluren, Transfluenzpässe sind noch weiter verbreitet, als unmittelbar wahrgenommen werden kann, da der sehr dichte und nur schwer zu durchdrin-

1) Die Fahrt wurde dankenswerterweise durch eine Sachbeihilfe der Deutschen Forschungsgemeinschaft und das Entgegenkommen von Prof. Dr. Hubert Miller, damals an der Universidad Austral de Chile in Valdivia, ermöglicht. 


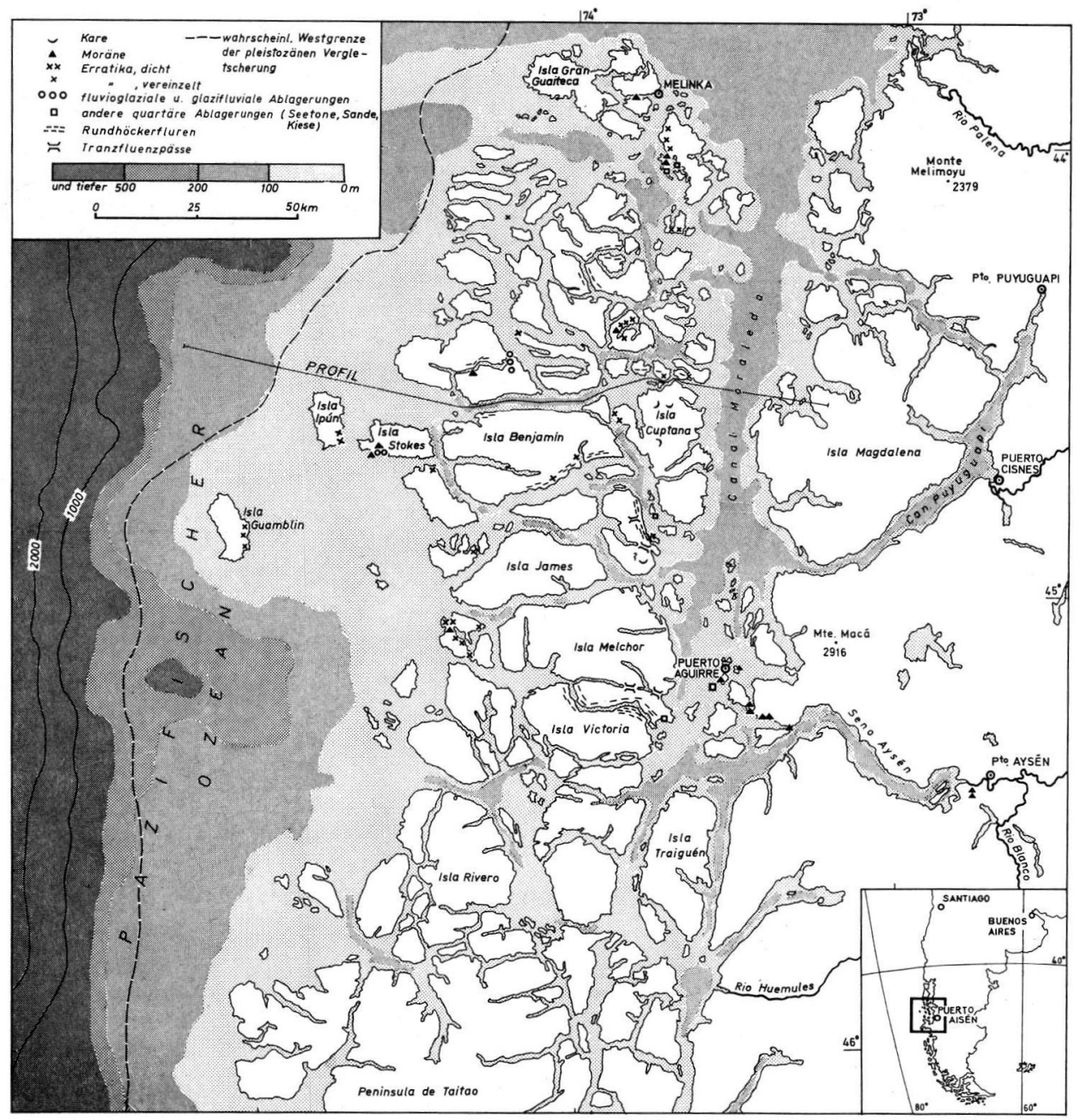

Abb. 1. Guaitecas-Inseln und Chonos-Archipel/Südchile.

gende immergrüne Wald vieles verhüllt. Besonders charakteristisch ist das ungleichsinnige Gefälle der Kanalböden, die, soweit sie bisher genauer ausgelotet sind, den Wechsel von Schwellenzonen und Becken (bis über $500 \mathrm{~m}$ tief) erkennen lassen (Abb. 2). Zum Außensaum des Archipels steigen die breiten Böden der Kanäle generell an und streichen auf einer nur 50 bis wenig über $100 \mathrm{~m}$ tiefen Schelfplattform aus. Wie vor den Mündungen

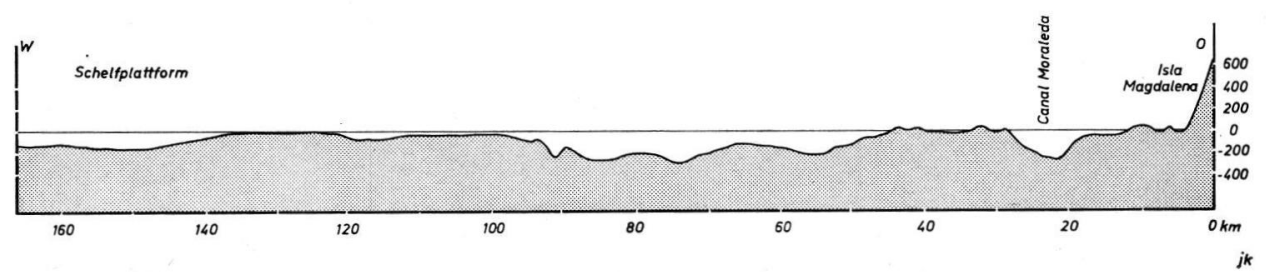

Abb. 2. Längsprofil durch den Canal King ( $44^{\circ} 30^{\prime}$ Süd), 15-fache Überhöhung. 
der skandinavischen Fjorde tritt nicht selten eine Klippenzone (strandflate) auf; die tiefen Rinnen, die den skandinavischen Schelf gliedern, fehlen aber hier. Typisch für Westpatagonien sind im Gegensatz zur norwegischen Fjordküste die zahlreichen Querkanäle und die vielen Inseln. WeIschet (1970) führt diese Unterschiede darauf zurück, daß in den Patagonischen Anden ein junges Kettengebirge, in Skandinavien aber ein stark abgetragenes Gebiet mit geringen Höhenunterschieden bei Eintritt der Vergletscherung vorlag. In Petagonien werden auch nacheiszeitliche Landsenkungen als Ursache dieses Formenbildes angenommen, während sich Skandinavien glazialeustatisch hebt.

Die Annahme einer postglazialen Senkung der Patagonischen Anden findet sich schon in der älteren Literatur, u. a. bei Gallois (1901), und hat sich bis heute behauptet. Dabei werden Absenkungswerte zwischen etwa $100 \mathrm{~m}$ (BRüGGEN 1950) und $1000 \mathrm{~m}$ (Arnold Heim 1951) vertreten, die besonders die Westseite des Gebirges betroffen haben sollen. Dadurch seien die Trogtäler und Endmoränen unter den Meeresspiegel getaucht worden. Ausgangspunkt für die Erklärung der Genese der Fjordküste und der Inselfluren ist also, wenn auch nicht immer deutlich ausgesprochen, eine Kippung des Gebirges.

Bei einer näheren Betrachtung ist keiner der Beweise für eine Kippung oder Senkung der Patagonischen Anden eindeutig. Bereits DARwIN (1846) hat nach seiner Fahrt mit der "Beagle“ entlang der Küste Westpatagoniens auf hochgelegene, horizontal verlaufende Strandterrassen aufmerksam gemacht. NordenskJÖld (1901 bzw. 1907) schilderte Ähnliches aus dem Umkreis der Magellan-Straße, und Halle (1910) erwähnte eine 50-m-Terrasse beiderseits des Fitz-Roy-Kanals zwischen Seno Skyring und Seno Otway. Auer (1957) hat sowohl bei seinen Mooruntersuchungen auf Feuerland als auch bei Verfolgen der Strandterrassen an der ostpatagonischen Küste ab Bahía Blanca keine generelle Landsenkung feststellen können. Gegen eine solche sprechen auch teilweise sehr hoch gelegene Terrassen (Abrasionsflächen?) im Chonos-Archipel. Auf den Inseln Churecue und Elena am Ausgang des Seno Aysén und auf dem Festland südlich dieser Inseln sind Verflachungen in 10, 30 und etwa $150 \mathrm{~m}$ ausgebildet. Die Inseln Tangbac und Teresa westlich des Canal Moraleda tragen in ca. $200 \mathrm{~m}$ Höhe schöne Flächen. Auf Leucayec und seinen Nachbarinseln in der Gruppe der Guaitecas-Inseln sind mehrere Niveaus ausgebildet: in 10, 20-25, 50-60 m und in höherer Lage. Auch auf den Inseln Melchor, Stokes, Jorge, Rowlett und Chalarayel sind Verflachungen zu beobachten, die teilweise glazial überprägt wurden. Bei einer speziellen Aufnahme der Inseln würden sich bestimmt weitere Terrassenreste finden, die keinen Hinweis auf Verstellungen geben.

Häufig werden die abgestorbenen oder absterbenden Wälder unter oder im Meeresspiegelniveau auf dem Landrücken von Ofqui, an der Bahía Esploradores und an der Mündung des Río Huemules in den Estero Elefantes als Anzeichen einer noch immer andauernden Landsenkung angesehen (STEFFEN 1913, 1919). Diese Wälder stocken ausnahmslos auf sehr jungen und sehr mächtigen, lockeren Aufschüttungen aus fluvioglazialem und vulkanischem Material, das in die übertieften Kanäle verfrachtet wurde. Die Senkungserscheinungen sind Folgen der Verfestigung (Diagenese) und der hiermit verbundenen Sackung der Alluvionen. Nirgends finden sich abgestorbene Wälder über festem Anstehendem.

Ebensowenig läßt sich aus den Oberflächenformen auf junge Landsenkung schließen, da auch Fjorde kein sicheres Anzeichen für diesen Vorgang sind, sondern lediglich der Beweis, daß die Übertiefung auch beachtlich unter den Meeresspiegel reichen kann. Dabei wird sicherlich die Ausrichtung von vielen Kanälen auf alte Talanlagen zurückgehen, deren Talboden vor der glazialen Erniedrigung wesentlich höher lag. Im übrigen lag die Ausgangsoberfläche für die Eisarbeit auf der Westseite des Gebirges wie heute auch im Pleistozän niedriger als auf der Ostseite, eine Folge der unterschiedlichen Erosions- und Abtragungsleistung der pazifischen und atlantischen Flüsse. 
Als Hinweis auf eine Kippung oder Senkung der Westseite der Patagonischen Anden wird die Verlagerung der Wasserscheide angesehen. Die Verlagerung der Wasserscheide geht jedoch auf rückschreitende Erosion und damit verbundene Anzapfungsvorgänge durch die kurzen, steilen Flüsse der überaus regenreichen pazifischen Seite zurück, die den langen gefällsarmen Flüssen der regenarmen atlantischen Seite mehr oder weniger große Teile des Einzugsgebietes raubten. So entstanden bereits präpleistozän die großen transandinen Quertäler. Eine Kippung wird auch dadurch widerlegt, daß in den östlichen Randanden bzw. am Ostrand des Gebirges miozäne oder unterpliozäne feinkörnige Serien und Konglomerate horizontal oder fast horizontal (mit schwachem O-Fallen) lagern bzw. entlang einer Flexur gebirgswärts aufgebogen sind. Bei einer Kippung wäre W-Fallen zu erwarten. Geomorphologische Kriterien sprechen ebenfalls gegen eine Kippung. Die wahrscheinlich mittelpliozänen Altflächen östlich der höchsten Erhebungen des Gebirges schließen sich, ohne Diskontinuitäten aufzuweisen, zu einer sanft ostwärts abdachenden Oberfläche zusammen, wie es einer alten Entwässerung entspricht. Ihre Ausbildung wäre bei größeren relativen Höhenunterschieden im Gebirge, also bei höherem Oberflächengefälle nicht möglich gewesen, sondern ein kräftig zertaltes Relief hätte sich eingestellt.

Den Beweis für eine postpleistozäne Kippung sieht Arnold Heim (1951) in der Identität von pleistozäner Eisscheide und einer einstmals westlicheren Lage der mittelpliozänen interozeanischen Wasserscheide. Dies kann insofern nicht überraschen, als sie in der Zone der höchsten Gipfel oder Gipfelgruppen des blockförmig zerlegten Gebirges verliefen.

Der Hinweis auf die große Ausdehnung der Moränen aller vier quartären Vergletscherungen auf der atlantischen Seite, denen die Aquivalente auf der Westseite des Gebirges $\mathrm{zu}$ fehlen scheinen und zu welchen die spärliche rezente Vergletscherung in keinem Verhältnis steht, ist ebensowenig stichhaltig. Wie der Vergleich zeigt, stehen holozäne und pleistozäne Ausdehnung der Gletscher in den Patagonischen Anden in dem gleichen Verhältnis, wie es sich auch für die Alpen darstellt. Das scheinbare Fehlen von Endmoränenlandschaften auf der Westseite geht auf drei Ursachen zurück: auf den postglazialen Meeresspiegelanstieg, der wegen der tiefen Lage der Landoberfläche zur Überflutung von Moränenlandschaften führte, zum anderen auf die Tatsache, daß zumindest im seichten Schelfgebiet durch die beständige Einwirkung des Ozeans eine Einebnung von Moränenwällen erfolgte (s. auch PAULSEN 1936) und in Gebieten größerer Meerestiefe die Gletscher in das Meer kalbten. Letztlich ist die geringe Kenntnis der Verbreitung pleistozäner Ablagerungen im pazifischen Patagonien eine Ursache für diese Auffassung.

Alle bisher angeführten Versuche, das „ertrunkene“ Tälerrelief als Folge einer Kippung des Gebirges zu deuten, führten nicht zu befriedigenden Ergebnissen. Auf der Fahrt in der Inselwelt des Chonos-Archipels gelang es, an zahlreichen Stellen neben den morphologischen Charakteristika weitere Hinweise auf die ehemalige Vergletscherung und deren Reichweite zu finden. So wird das Auftreten von Erratika von $\mathrm{O}$ nach $\mathrm{W}$ häufiger. Besonders dichte Vorkommen fanden sich in den inneren Kanälen und an der Westküste der Insel Kent (westlich der Insel Melchor). Dabei handelt es sich vorwiegend um Granit- und Quarzitblöcke, die aus der Zentralzone des Gebirges stammen, während die Vulkanite aus dem Gebiet östlich der mittelpliozänen Wasserscheide und der Eisscheide bezeichnenderweise fehlen.

Auch an der Küste der Insel Stokes nehmen die Erratika von O nach W stark zu. Im niedrigen SW-Teil der Insel ist an der Südküste über rund $3 \mathrm{~km}$ eine bis $40 \mathrm{~m}$ mächtige Ablagerung aufgeschlossen, die wegen der schlechten Klassierung, der undeutlichen Schichtung und der geringen Zurundung der Einzelkomponenten und der sehr bunten Zusammensetzung als glazifluvial anzusprechen ist. Sie wird diskordant überdeckt von einer 2-5 m mächtigen, stärker verfestigten Grundmoräne, die das gesamte Relief der Halbinsel im SW von Stokes prägt. Zugleich tritt im Kanal südlich von Stokes gegen den 
Ozean hin eine Ausgangsschwelle auf, die mit zahlreichen Klippen besetzt ist. Die Kombination dieser Erscheinungen, die nachlassende Übertiefung anzeigt, spricht für die Nähe der Westgrenze der Eismassen.

Die Bestätigung dieser Annahme findet sich auf der weiter im W gelegenen Insel Ipún. An ihrer Küste (speziell um P to Scotchwell) liegt auf der Strandplattform bzw. auf den Strandwällen sehr viel ortsfremdes Material. Eine Verdriftung durch Eis ist ausgeschlossen, da wegen des niedrigeren Meeresspiegels während der Kaltzeiten das überaus seichte Schelfgebiet um die Insel Ipún trockengefallen sein muß. Nordöstlich des P to Scotchwell ist an der Steilküste über dem Tertiär, das die Insel aufbaut, erneut Moräne aufgeschlossen. Die in diese Bucht mündenden Flüsse bringen kantiges, allenfalls kantengerundetes, buntes Material - aufgearbeitete Moräne - aus dem Inneren. Eine Auswertung der Luftbilder von Ipún ergibt, daß die Oberfläche von annähernd N-S-streichenden Höhenrücken gebildet wird, die nach dem Material der Flüsse als Endmoränen gedeutet werden können. Entsprechende Verhältnisse wie an der Küste und im Inneren von Ipún liegen auch bei der Insel Guamblin vor.

Die überaus seichte und unzerschnittene Schelfplattform im N von Ipún und im Umkreis von Guamblin ist ein weiterer Hinweis für eine durch Abrasion eingeebnete Endmoränenlandschaft. Auf den Seekarten des Instituto Hidrográfico der chilenischen Armee sind für die Lotungsstellen überwiegend Steine, Sand und Kies als Untergrund angegeben. Interessant sind allerdings die Tiefenverhältnisse am Ausgang des Canal King zwischen der Insel Stokes im S und der Insel Level im N. Nach dem bis über $300 \mathrm{~m}$ tiefen mittleren Kanalstück wird der Fjord nach W bei zunehmender Verbreiterung immer flacher und verliert sich schließlich auf dem nicht einmal $50 \mathrm{~m}$ tiefen Schelf nördlich Ipún. Die Tiefenverhältnisse zeichnen hier ein typisches Zungenbecken nach.

Mit diesen Ergebnissen läßt sich für den Chonos-Archipel die Reichweite der pleistozänen Vergletscherung annähernd fixieren. Es muß im Gebiet der beiden Inseln und der nördlich und südlich anschließenden Schelfplattform sein westliches Ende erreicht haben. Das Eis reichte also im Vergleich zu Chiloä ungefähr $80-90 \mathrm{~km}$ weiter nach W, wie es bei der polnäheren Lage zu erwarten ist.

Der Verlauf der Eisrandlagen im N des Chonos-Archipels und der Guaitecas-Inseln und südlich Guamblin ist wegen unzureichender Kenntnis der feineren Formen und des Baumaterials des Schelfes nur mit Vorbehalten zu rekonstruieren. Ein Versuch wurde auf Abb. 1 gemacht. Als Anhaltspunkte dienten im $\mathrm{N}$ des Archipels die Verbreitung von Erratika und ein Moränenvorkommen auf einer kleinen Insel im Canal Lagreze westlich Melinka (Gonra). Im S muß der Verlauf zunächst hypothetisch bleiben.

Östlich der ehemaligen Eisrandlage konnten weitere Moränenvorkommen, fluvioglaziale und glazifluviale Ablagerungen gefunden werden. In der Guaitecas-Gruppe zeichnen sich besonders die Insel Leucayec und die ihr westlich vorgelagerten kleinen Inseln nördlich der Isla Ovalada durch solche Sedimente aus. Unter einer 2-3 m mächtigen Grundmoränendecke liegen gestauchte glazifluviale Tone, Sande und Kiese bis zu $5 \mathrm{~m}$ Mächtigkeit. Größere Ausdehnung haben glaziale und fluvioglaziale Ablagerungen auch auf den Inseln um Puerto Aguirre. Nördlich dieses Ortes (um den Flugplatz) existieren ausgedehnte Moränendecken und unmittelbar im $\mathrm{N}$ des Hafens ist an einem Kliff eine Deltaschüttung aus fluvioglazialem Material aufgeschlossen. Ebenso wie die Seetone wenig westlich des Puerto Lagunas im S der Insel Melchor sind sie wahrscheinlich als Ablagerungen in Eisstauseen gegen Ende der Würmvereisung anzusehen, als bereits ein Eiszerfall einsetzte. Einem Rückzugsstadium des Würmeises oder einem stadialen Vorstoß entsprechen die Endmoränen, die die ehemalige Insel Elisa nördlich des Ausganges des Seno Aysén mit dem Festland verbinden und die offenbar wenig weiter östlich als submarine Schwelle den Canal Pilcomayo vom Seno oder Fjordo Aysén trennen. 
Als einzige Insel im gesamten Chonos-Archipel dürfte wegen ihrer Höhe die Insel Cuptana $(1640 \mathrm{~m})$ eine nennenswerte pleistozäne Eigenvergletscherung entwickelt haben. In der Gipfelregion dieser Insel sind jedenfalls Kare entwickelt. Zwar fehlen karähnliche Formen auf anderen, bis 700 oder $800 \mathrm{~m}$ aufragenden Insel nicht, doch läßt sich ihre glaziale Entstehung nicht mit Sicherheit vertreten.

\section{Literatur}

Auer, V.: Über die eustatische Bewegung des Meeresspiegels in Feuerland-Patagonien und anschließende Fragen. - Stuttgarter Geogr. Studien 69, 407-418, Hermann-Lautensach-Festschrift, Stuttgart 1957.

BrüGGEN, J.: Zur Glazialgeologie der chilenischen Anden. - Geol. Rdsch. 20, 1-35, 4 Abb., Berlin 1929.

— : Fundamentos de la Geologia de Chile. - Santiago de Chile 1950.

Caldenius, C.: Los glaciaciones cuarternarias en la Patagonia y Tierra del Fuego. - Geografiska Annaler 14, 1-164, Stockholm 1932.

Czajka, W.: Die Reichweite der pleistozänen Vereisung Patagoniens. — Geol. Rdsch. 45, 634-686, 24 Abb., 1 Taf., Stuttgart 1957.

Darwin, C.: Geological observations on South America. London 1846; deutsch unter dem Titel: Geologische Beobachtungen über Südamerika und kleinere geologische Abhandlungen. Stuttgart 1890.

Gallors, L.: Les Andes de Patagonie. - Annales de Geographie 10, 232-259, Paris 1901.

Halle, Th. G.: On Quarternary deposits and changes of level in Patagonia and Tierra del Fuego. - Bull. geol. Inst. Uppsala 9, 93-119, Uppsala 1910.

Heim, A.: On the Glaciation of South America as related to Tectonics. Observations 1939-1947. - Ecl. Geol. Helv. 44, 171-182, 3 Abb., Basel 1951.

NoRdenskjöLD, O.: Die Landschaftsformen der Magellan-Länder mit besonderer Rücksicht auf die glacialen Bildungen. - Verh. 7. Int. Geogr. Kongr. Berlin 1899, 2, 303-306,Berlin 1901.

- : Über die posttertiären Ablagerungen der Magellan-Länder. In: Wissenschaftliche Ergebnisse der schwedischen Expedition nach den Magellan-Ländern 1895-1987. Bd. I, Nr. 2, Stockholm 1907.

Paulsen, I.: Das südchilenische Fjordgebiet. Untersuchung der untermeerischen Teile. - Archiv deutsch. Seewarte 56, Nr. 4, Hamburg 1936.

Steffen, H.: Die Landbrücke von Ofqui in Westpatagonien. - Mitt. Geogr. Ges. für Thüringen zu Jena 31, 19-64, Jena 1913.

— : Westpatagonien. 2 Bde., Berlin 1919.

Weischet, W.: Chile. Seine länderkundliche Individualität und Struktur. Darmstadt 1970.

Karten des Instituto Hidrográficode la Armada de Chile

$\begin{array}{ll}718 & \text { Islas Guaitecas } \\ 801 & \text { Canal Moraleda, Islas Guaitecas a Puerto Lagunas } \\ 802 & \text { Canales Puyuguapi y Jacaf } \\ 803 & \text { Canales Perez Norte y Perez Sur } \\ 804 & \text { Puertos en el Canal Moraleda } \\ 805 & \text { Puerto Lagunas y Bahía Anna Pink } \\ 808 & \text { Croquis de Puertos en el Archipiélago de Los Chonos } \\ 809 & \text { Puerto Lagunas a Pta Pescadores } \\ 810 & \text { Canal Ferronave } \\ 811 & \text { Bahía Chacabuco y Rio Aysén } \\ 812 & \text { Pta Pescadores, Istmo de Ofqui } \\ 815 & \text { Bahía Anna Pink }\end{array}$

$1: 100000$

$1: 250000$

$1: 250000$

$1: 125000$

Puertos en el Canal Moraleda

Puerto Lagunas y Bahía Anna Pink

$1: 150000$

Puerto Lagunas a Pta Pescadores

$1: 150000$

$1: 60000$

$1: 10000$

$1: 150000$

$1: 300000$

Manuskript eingeg. 21. 1. 1974

Anschrift des Verf.: Prof. Dr. K. Fischer, Lehrstuhl für Physische Geographie, Universität Augsburg, 89 Augsburg, Alter Postweg 101. 